\title{
A State Estimation Method for Sound Environment System with Unknown Observation Mechanism by Introducing Fuzzy Inference
}

\author{
Hisako Orimoto, Akira Ikuta \\ Department of Management Information Systems, Prefectural University of Hiroshima, Hiroshima, Japan \\ Email: \{orimoto, ikuta\}@pu-hiroshima.ac.jp
}

Received January 21, 2012; revised April 23, 2012; accepted June 14, 2012

\begin{abstract}
The observed phenomena in real sound environment system often contain uncertainty such as the additional external noise with unknown statistics. Furthermore, there is complex nonlinear relationship between the specific signal and the observations, and it cannot be exactly expressed in any definite functional form. In these situations, it is one of reasonable analysis methods to treat the objective sound environment system as a fuzzy system. In this study, a state estimation method for a specific signal under the existence of an unknown observation mechanism and external noise of unknown statistics is proposed by introducing fuzzy inference. The effectiveness of the proposed theoretical method is experimentally confirmed by applying it to the actually observed data in the sound environment.
\end{abstract}

Keywords: State Estimation; Sound Environment System; Unknown Observation Mechanism; Fuzzy Inference

\section{Introduction}

The observation data in actual sound environment system exhibit various types of fluctuation characteristics, and these often contain uncertainty. For example, the observed signal is inevitably contaminated by the concurrent external noise (i.e., background noise) of arbitrary distribution type of unknown statistics. In this situation, in order to evaluate the specific signal based on the observed noisy data, it is indispensable to introduce some unified state estimation methods adaptable to various uncertainty caused by complexity, diversity and unknown property existing in the actual sound environment systems.

Though several state estimation methods have been proposed up to now, these state estimation algorithms have been realized by introducing the additive model of the specific signal and the external noise under an assumption of known statistics of the external noise [1-8]. On the other hand, it is necessary to pay our attention on the fact that the observation data in the sound environment system are often contain uncertainty due to several causes. For example, the observation in sound environment system can be generally expressed in an additive model of the specific signal and the background noise on energy scale by using the additive property of acoustic energy. However, for an assessment and a countermeasure of the sound environment, several evaluation quanti- ties such as $L_{e q}$ (averaged energy on a decibel scale), $L_{x}((100-x)$ percentile level, $x=5,10,50,90,95)$ and $L_{\max }$ (maximum level), etc. for the specific signal, have to be considered. There are complex nonlinear and uncertain relations between the noise evaluation quantities of the specific signal and the background noise, and it is difficult in general to find the functional relationship between them. Furthermore, it is actually difficult to know in advance statistical properties of the background noise. Therefore, in order to remove effects of the background noise from the observed evaluation quantities under existence of the background noise, standard state estimation method based on an additive model of the specific signal and the background noise of known statistics cannot be applied. In this situation, the relationship between the observed evaluation quantities and the background noise has to be generally considered as a system model with unknown observation mechanism. In our previous study, a method for estimating the specific signal for the sound environment system with uncertainty has been proposed by adopting the conditional probability distribution reflecting the correlation information between the specific signal and observation as the system characteristics [9]. Though the method proposed previously can be applied effectively to actual complex sound environmental system with uncertainty, it needs a lot of priori information on the statistics of the specific signal and observations. 
In this study, a practical state estimation method for sound environment systems with unknown observation mechanism of contamination by the background noise of unknown statistics is proposed by introducing the fuzzy inference in a different form the previous methods. More specifically, a membership function is first introduced for the unknown mechanism of a sound environment system. Next, by applying a fuzzy inference for the relationship between the observed data (i.e., evaluation quantities observed under the existence of a background noise) and the state variable (i.e., evaluation quantities of the specific signal), a state estimation algorithm is derived, based on Bayes' theorem as the fundamental principle of the estimation in the similar manner to the previously reported estimation method [4,5]. The effectiveness of the proposed method is experimentally confirmed by applying it to the estimation of evaluation quantities for road traffic noise under existence of a background noise.

\section{State Estimation for Sound Environment System with Unknown Observation Mechanism}

\subsection{Formulation of Sound Environment System by Introducing Fuzzy Inference}

Let $x_{k}$ and $y_{k}$ be state variable and observation at a discrete time $k$ for sound environment systems. It is assumed that the mutual relationship between $x_{k}$ and $y_{k}$ is unknown. For example, the observations in sound environment are inevitably contaminated by the external noise (i.e., background noise) of arbitrary distribution type. In general, by using the additive property of acoustic energy, the observation energy under the existence of external noise can be expressed in an additive model of the specific signal and the background noises in energy scale. However, for the stochastic evaluation quantities: $L_{e q}$ and $L_{x}$ which are used in the evaluation of actual sound environment, there are complex nonlinear relations between the evaluation quantities of the specific signal and the observed evaluation quantities. For an assessment of sound environment, it is often necessary to investigate the fluctuation of evaluation quantities for the specific signal. Therefore, the evaluation quantities of the specific signal at the $k$-th short time interval and the observed evaluation quantities at the $k$-th time interval have to be regarded as the unknown state variable $x_{k}$ and the observation $y_{k}$ respectively. Since it is difficult in general to find functional relationship between the state variable $x_{k}$ and the observation $y_{k}$, the relationship between $x_{k}$ and $y_{k}$ has to be considered as a sound environment system with unknown observation mechanism. In this study, the following IF-THEN rule is introduced for the unknown mechanism of the sound envi- ronment system:

$$
\begin{aligned}
& \text { Rule 1: IF } x_{k} \text { is } A_{1} \text { THEN } y_{k} \text { is } B_{1} \\
& \text { Rule 2: IF } x_{k} \text { is } A_{2} \text { THEN } y_{k} \text { is } B_{2} \\
& \ldots \\
& \text { Rule } N: \text { IF } x_{k} \text { is } A_{N} \text { THEN } y_{k} \text { is } B_{N},
\end{aligned}
$$

where $A_{i}$ and $B_{i}(i=1,2, \cdots, N)$ denote fuzzy sets corresponding to the several local state spaces of $x_{k}$ and $y_{k}$, and have membership functions $\mu_{A_{i}}\left(x_{k}\right)$ and $\mu_{B_{i}}\left(y_{k}\right)(i=1,2, \cdots, N)$. For example, $A_{1}, A_{2}, \cdots, A_{N}$ are fuzzy sets describing the local $N$ regions with vague borders such as "very low level", "low level", ..., "very high level", for the fluctuation range of the sound level $x_{k}$. Applying fuzzy inference to (1), $y_{k}$ is given by [10]

$$
y_{k}=\frac{\sum_{i=1}^{N} \bar{y}_{i} \mu_{A_{i}}\left(x_{k}\right)}{\sum_{i=1}^{N} \mu_{A_{i}}\left(x_{k}\right)}
$$

where $\bar{y}_{i}$ is the value at which the membership function $\mu_{B_{i}}\left(y_{k}\right)$ achieved its maximum value. Furthermore, as the membership function $\mu_{A_{i}}\left(x_{k}\right)$, the Gaussian type, defined by [10]

$$
\mu_{A_{i}}\left(x_{k}\right)=\exp \left\{-\frac{1}{2}\left(\frac{x_{k}-\bar{x}_{i}}{\sigma_{i}}\right)^{2}\right\}
$$

where $\bar{x}_{i}$ and $\sigma_{i}$ are parameters, is adopted.

In the next section, an estimation method for the state variable $x_{k}$ of the specific signal based on the recursive observation $y_{k}$ is derived. Though the parameter $\sigma_{i}$ in (3) can be generally given based on the prior information (or, through trial and error), it can be regarded as unknown constant parameter $\alpha\left(=\sigma_{i}\right.$ for every $\left.i\right)$ and estimated simultaneously with the state variable $x_{k}$ by introducing the following simple dynamic model:

$$
\alpha_{k+1}=\alpha_{k} \text {. }
$$

\subsection{Estimation Algorithm by Introducing Bayes' Theorem}

In order to derive an estimation algorithm for a state variable $x_{k}$, with an arbitrary distribution, we focus our attention on Bayes' theorem for the conditional probability density function (abbr. pdf). Since the parameter $\alpha_{k}$ is also unknown, the conditional pdf of $x_{k}$ and $\alpha_{k}$ must be considered.

$$
P\left(x_{k}, \alpha_{k} \mid Y_{k}\right)=\frac{P\left(x_{k}, \alpha_{k}, y_{k} \mid Y_{k-1}\right)}{P\left(y_{k} \mid Y_{k-1}\right)}
$$

where $Y_{k}\left(=\left\{y_{1}, y_{2}, \cdots y_{k}\right\}\right)$ is a set of observation data up to $k$-th time. The conditional joint pdf of the state variable $x_{k}$, the parameter $\alpha_{k}$ and the observation $y_{k}$ 
in (5) can be generally expanded in a statistical orthogonal expansion series $[4,5]$ :

$$
\begin{aligned}
& P\left(x_{k}, \alpha_{k}, y_{k} \mid Y_{k-1}\right) \\
& =P_{0}\left(x_{k} \mid Y_{k-1}\right) P_{0}\left(\alpha_{k} \mid Y_{k-1}\right) P_{0}\left(y_{k} \mid Y_{k-1}\right) \\
& \sum_{l=0}^{\infty} \sum_{m=0}^{\infty} \sum_{n=0}^{\infty} A_{l m n} \varphi_{l}^{(1)}\left(x_{k}\right) \varphi_{m}^{(2)}\left(\alpha_{k}\right) \varphi_{n}^{(3)}\left(y_{k}\right)
\end{aligned}
$$

After substituting (6) into (5), taking the conditional expectation of the function $f_{N_{1}, N_{2}}\left(x_{k}, \alpha_{k}\right)$ and using the orthonormal condition for the function $\varphi_{l}^{(1)}\left(x_{k}\right)$ and $\varphi_{m}^{(2)}\left(\alpha_{k}\right)$, through a similar calculation process to that used in a previously reported estimation method [4,5], the estimate of an arbitrary polynomial function

$f_{N_{1}, N_{2}}\left(x_{k}, \alpha_{k}\right)$ of $x_{k}$ and $\alpha_{k}$ of $N\left(\equiv\left(N_{1}, N_{2}\right)\right)$-th order can be derived in an infinite series expression, as follows:

$$
\begin{aligned}
& \hat{f}_{N_{1}, N_{2}}\left(x_{k}, \alpha_{k}\right) \equiv\left\langle f_{N_{1}, N_{2}}\left(x_{k}, \alpha_{k}\right) \mid Y_{k}\right\rangle \\
& =\frac{\sum_{l=0}^{N_{1}} \sum_{m=0}^{N_{2}} \sum_{n=0}^{\infty} C_{l m}^{N_{1} N_{2}} A_{l m n} \varphi_{n}^{(3)}\left(y_{k}\right)}{\sum_{n=0}^{\infty} A_{00 n} \varphi_{n}^{(3)}\left(y_{k}\right)}
\end{aligned}
$$

with

$$
A_{l m n} \equiv\left\langle\varphi_{l}^{(1)}\left(x_{k}\right) \varphi_{m}^{(2)}\left(\alpha_{k}\right) \varphi_{n}^{(3)}\left(y_{k}\right) \mid Y_{k-1}\right\rangle
$$

The three functions $\varphi_{l}^{(1)}\left(x_{k}\right), \varphi_{m}^{(2)}\left(\alpha_{k}\right)$ and $\varphi_{n}^{(3)}\left(y_{k}\right)$ are the orthonormal polynomials of degrees $l, m$ and $n$, with weighting functions $P_{0}\left(x_{k} \mid Y_{k-1}\right), P_{0}\left(\alpha_{k} \mid Y_{k-1}\right)$ and $P_{0}\left(y_{k} \mid Y_{k-1}\right)$, which can be artificially chosen as the pdfs describing the above dominant parts of the actual fluctuation, or as well-known standard pdfs such as Gaussian or Gamma distribution functions. All the coefficients $C_{l m}^{N_{1} N_{2}}$ are appropriate constants in the case when the function $f_{N_{1}, N_{2}}\left(x_{k}, \alpha_{k}\right)$ is expressed in a series expansion form using $\varphi_{l}^{(1)}\left(x_{k}\right)$ and $\varphi_{m}^{(2)}\left(\alpha_{k}\right)$ :

$$
f_{N_{1}, N_{2}}\left(x_{k}, \alpha_{k}\right)=\sum_{l=0}^{N_{1}} \sum_{m=0}^{N_{2}} C_{l m}^{N_{1} N_{2}} \varphi_{l}^{(1)}\left(x_{k}\right) \varphi_{m}^{(2)}\left(\alpha_{k}\right)
$$

As a concrete example of a standard pdf, the wellknown Gaussian distribution is adopted:

$$
\begin{aligned}
& P_{0}\left(x_{k} \mid Y_{k-1}\right)=N\left(x_{k} ; x_{k}^{*}, \Gamma_{x_{k}}\right) \\
& P_{0}\left(\alpha_{k} \mid Y_{k-1}\right)=N\left(\alpha_{k} ; \alpha_{k}^{*}, \Gamma_{\alpha_{k}}\right) \\
& P_{0}\left(y_{k} \mid Y_{k-1}\right)=N\left(y_{k} ; y_{k}^{*}, \Gamma_{y_{k}}\right)
\end{aligned}
$$

with

$$
\begin{gathered}
N\left(x ; \mu, \sigma^{2}\right) \equiv \frac{1}{\sqrt{2 \pi \sigma^{2}}} \exp \left\{-\frac{(x-\mu)^{2}}{2 \sigma^{2}}\right\}, \\
x_{k}^{*} \equiv\left\langle x_{k} \mid Y_{k-1}\right\rangle, \Gamma_{x_{k}} \equiv\left\langle\left(x_{k}-x_{k}^{*}\right)^{2} \mid Y_{k-1}\right\rangle \\
a_{k}^{*} \equiv\left\langle a_{k} \mid Y_{k-1}\right\rangle, \Gamma_{a_{k}} \equiv\left\langle\left(a_{k}-a_{k}^{*}\right)^{2} \mid Y_{k-1}\right\rangle \\
y_{k}^{*} \equiv\left\langle y_{k} \mid Y_{k-1}\right\rangle, \Gamma_{y_{k}} \equiv\left\langle\left(y_{k}-y_{k}^{*}\right)^{2} \mid Y_{k-1}\right\rangle .
\end{gathered}
$$

Then, the orthonormal polynomials with three weighting pdfs in (10)-(12) can be given in terms of the wellknown Hermite polynomials [11].

$$
\begin{aligned}
& \varphi_{l}^{(1)}\left(x_{k}\right)=\frac{1}{\sqrt{l} !} H_{l}\left(\frac{x_{k}-x_{k}^{*}}{\sqrt{\Gamma_{x_{k}}}}\right) \\
& \varphi_{m}^{(2)}\left(\alpha_{k}\right)=\frac{1}{\sqrt{m !}} H_{m}\left(\frac{\alpha_{k}-\alpha_{k}^{*}}{\sqrt{\Gamma_{\alpha_{k}}}}\right) \\
& \varphi_{n}^{(3)}\left(y_{k}\right)=\frac{1}{\sqrt{n !}} H_{n}\left(\frac{y_{k}-y_{k}^{*}}{\sqrt{\Gamma_{y_{k}}}}\right)
\end{aligned}
$$

Using (2) and (3), the two variables $y_{k}^{*}$ and $\Gamma_{y_{k}}$ in (13) can be expressed as:

$$
\begin{aligned}
& y_{k}^{*}=\left\langle\sum_{i=1}^{N} \bar{y}_{i} \exp \left\{-\frac{1}{2}\left(\frac{x_{k}-\bar{x}_{i}}{\alpha_{k}}\right)^{2}\right\} / \sum_{i=1}^{N} \exp \left\{-\frac{1}{2}\left(\frac{x_{k}-\bar{x}_{i}}{\alpha_{k}}\right)^{2}\right\} \mid Y_{k-1}\right\rangle \\
& \Gamma_{y_{k}}=\left\langle\left[\sum_{i=1}^{N} \bar{y}_{i} \exp \left\{-\frac{1}{2}\left(\frac{x_{k}-\bar{x}_{i}}{\alpha_{k}}\right)^{2}\right\} / \sum_{i=1}^{N} \exp \left\{-\frac{1}{2}\left(\frac{x_{k}-\bar{x}_{i}}{\alpha_{k}}\right)^{2}\right\}-y_{k}^{*}\right]^{2} \mid Y_{k-1}\right\rangle
\end{aligned}
$$

Furthermore, each expansion coefficient $A_{l m n}$ defined by (8) can be expressed as

$$
A_{l m n}=\left\langle\frac{1}{\sqrt{l} !} H_{l}\left(\frac{x_{k}-x_{k}^{*}}{\sqrt{\Gamma_{x_{k}}}}\right) \frac{1}{\sqrt{m !}} H_{m}\left(\frac{\alpha_{k}-\alpha_{k}^{*}}{\sqrt{\Gamma_{\alpha_{k}}}}\right) \frac{1}{\sqrt{n !}} H_{n}\left(\frac{\sum_{i=1}^{N} \bar{y}_{i} \exp \left\{-\frac{1}{2}\left(\frac{x_{k}-\bar{x}_{i}}{\alpha_{k}}\right)^{2}\right\} / \sum_{i=1}^{N} \exp \left\{-\frac{1}{2}\left(\frac{x_{k}-\bar{x}_{i}}{\alpha_{k}}\right)^{2}\right\}-y_{k}^{*}}{\sqrt{\Gamma_{y_{k}}}}\right) \mid Y_{k-1}\right\rangle
$$


The variables $y_{k}^{*}, \Gamma_{y_{k}}$ and the expansion coefficient $A_{t m n}$ in (17), (18) and (19) are given by the predictions of $x_{k}$ and $\alpha_{k}$ at a discrete time $k-1$ (i.e., the expectation value of arbitrary functions of $x_{k}$ and $\alpha_{k}$ conditioned by $\left.Y_{k-1}\right)$. Consequently, the unified algorithm for estimating the state variable can be obtained. In two special cases when $f_{1,0}\left(x_{k}, \alpha_{k}\right)=x_{k}$ and

$f_{2,0}\left(x_{k}, \alpha_{k}\right)=\left(x_{k}-\hat{x}\right)^{2}$, estimates related to mean and variance of the state variable are expressed as follows:

$$
\begin{aligned}
& \hat{x}_{k} \equiv\left\langle x_{k} \mid Y_{k}\right\rangle \\
&= \frac{\sum_{n=0}^{\infty}\left\{C_{00}^{10} A_{00 n}+C_{10}^{10} A_{10 n}\right\} \frac{1}{\sqrt{n !}} H_{n}\left(\frac{y_{k}-y_{k}^{*}}{\sqrt{\Gamma_{y_{k}}}}\right)}{\sum_{n=0}^{\infty} A_{00 n} \frac{1}{\sqrt{n !}} H_{n}\left(\frac{y_{k}-y_{k}^{*}}{\sqrt{\Gamma_{y_{k}}}}\right)} \\
& P_{x_{k}} \equiv\left\langle\left(x_{k}-\hat{x}_{k}\right)^{2} \mid Y_{k}\right\rangle \\
&=\frac{\sum_{n=0}^{\infty}\left\{C_{00}^{20} A_{00 n}+C_{10}^{20} A_{10 n}+C_{20}^{20} A_{20 n}\right\} \frac{1}{\sqrt{n !}} H_{n}\left(\frac{y_{k}-y_{k}^{*}}{\sqrt{\Gamma_{y_{k}}}}\right)}{\sum_{n=0}^{\infty} A_{00 n} \frac{1}{\sqrt{n !}} H_{n}\left(\frac{y_{k}-y_{k}^{*}}{\sqrt{\Gamma_{y_{k}}}}\right)}
\end{aligned}
$$

with

$$
\begin{aligned}
& C_{00}^{10} \equiv x_{k}^{*}, C_{10}^{10} \equiv \Gamma_{x_{k}}, C_{00}^{20} \equiv \Gamma_{x_{k}}+\left(x_{k}^{*}-\hat{x}_{k}\right)^{2} \\
& C_{10}^{20} \equiv 2 \sqrt{\Gamma_{x_{k}}}\left(x_{k}^{*}-\hat{x}_{k}\right), C_{20}^{20} \equiv \sqrt{2} \Gamma_{x_{k}}
\end{aligned}
$$

Furthermore, by focusing on a polynomial function:

$$
f_{N_{1}, 0}\left(x_{k}-\alpha_{k}\right)=\frac{1}{\sqrt{N !}} H_{N_{1}}\left(\frac{x_{k}-\hat{x}_{k}}{\sqrt{P_{x_{k}}}}\right)
$$

the estimate for the expansion coefficient reflecting the non-Gaussian property of the state variable is given by Equation (24), where $C_{l 0}^{N_{1} 0} \quad\left(l=0,1, \cdots, N_{1}\right)$ are coefficients satisfying the following relationship:

$$
\frac{1}{\sqrt{N_{1} !}} H_{N_{1}}\left(\frac{x_{k}-\hat{x}_{k}}{\sqrt{P_{x_{k}}}}\right)=\sum_{l=0}^{N_{1}} C_{l 0}^{N_{1} 0} \frac{1}{\sqrt{l !}} H_{l}\left(\frac{x_{k}-x_{k}^{*}}{\sqrt{\Gamma_{x_{k}}}}\right)
$$

Finally, in order to derive the prediction step necessary to perform the recurrence estimation, the fuzzy inference is introduced again. More specifically, after dividing the fluctuation range of $x_{k}$ into $M$ fuzzy regions $S_{i}(i=$
$1,2, \cdots, M)$ with membership functions $\mu_{i}\left(x_{k}\right) \quad(i=1,2$, $\cdots, M)$, by creating fuzzy rules from given data sets, the following IF-THEN rules are obtained.

Rule 1: IF $x_{k}$ is $S_{1}$ THEN $x_{k+1}$ is $S_{1}^{\prime}$

Rule 2: IF $x_{k}$ is $S_{2}$ THEN $x_{k+1}$ is $S_{2}^{\prime}$

Rule $M$ : IF $x_{k}$ is $S_{M}$ THEN $x_{k+1}$ is $S_{M}^{\prime}$,

where $S_{1}^{\prime}$ is one of $M$ fuzzy sets $S_{i}(i=1,2, \cdots, M)$, and is decided adequately based on the prior information or measured data. For example, after evaluating two degrees for conformity of data pair $x_{k}$ and $x_{k+1}$ to $S_{i}$ and $S_{i}^{\prime}$, by selecting $S_{i}^{\prime}$ so as to maximize the synthesized degree of these two degrees, each rule of (26) can be assigned. By applying the center average defuzzification formula [12] to (26), a state transition model can be determined as

$$
\begin{gathered}
x_{k+1}=\frac{\sum_{i=1}^{M} \tilde{x}_{i} \mu_{i}\left(x_{k}\right)}{\sum_{i=1}^{M} \mu_{i}\left(x_{k}\right)} \\
\mu_{i}\left(x_{k}\right)=\exp \left\{-\frac{1}{2}\left(\frac{x_{k}-\tilde{x}_{i}}{\sigma}\right)^{2}\right\},
\end{gathered}
$$

where $\tilde{x}_{i}$ is the value at which the membership function $\mu_{i}\left(x_{k}\right)$ achieved its maximum value, and $\sigma$ is a constant parameter. By considering (4) and (27), the prediction algorithm can be given for an arbitrary polynomial function $g_{r}\left(x_{k+1}, \alpha_{k+1}\right)$ of $x_{k+1}$ and $\alpha_{k+1}$ of $r\left(\equiv\left(r_{1}, r_{2}\right)\right)$-th order, as follows:

$$
\begin{aligned}
& g_{r}^{*}\left(x_{k+1}, \alpha_{k+1}\right) \\
\equiv & \left\langle g_{r}\left(x_{k+1}, \alpha_{k+1}\right) \mid Y_{k}\right\rangle \\
= & \left\langle g_{r}\left(\sum_{i=1}^{M} \tilde{x}_{i} \mu_{i}\left(x_{k}\right)\right) / \sum_{i=1}^{M}\left(\mu_{i}\left(x_{k}\right), \alpha_{k}\right) \mid Y_{k}\right\rangle
\end{aligned}
$$

The above prediction can be evaluated by the estimates at a discrete time $k$. Therefore, by combining (7) with (29), the recurrence estimation of $x_{k}$ and $\alpha_{k}$ can be achieved.

\section{Application to Evaluation Quantities of Road Traffic Noise}

Finally, complete content and organizational editing be-

$$
\hat{a}_{N_{1}} \equiv \frac{1}{\sqrt{N_{1} !}}\left\langle H_{N_{1}}\left(\frac{x_{k}-\hat{x}_{k}}{\sqrt{P_{x_{k}}}}\right) \mid Y_{k}\right\rangle=\frac{\sum_{n=0}^{\infty}\left\{C_{00}^{N_{1} 0} A_{00 n}+C_{10}^{N_{1} 0} A_{10 n}+\cdots+C_{N_{1} 0}^{N_{1} 0} A_{N_{1} 0 n}\right\} \frac{1}{\sqrt{n !}} H_{n}\left(\frac{y_{k}-y_{k}^{*}}{\sqrt{\Gamma_{y_{k}}}}\right)}{\sum_{n=0}^{\infty} A_{00 n} \frac{1}{\sqrt{n !}} H_{n}\left(\frac{y_{k}-y_{k}^{*}}{\sqrt{\Gamma_{y_{k}}}}\right)}
$$


fore formatting. Please take note of the following items when proofreading spelling and grammar:

In order to confirm experimentally the validity of the proposed method, it is applied to the actually observed data in the sound environment. As the specific signal, the road traffic noise becoming one of the aggravating environmental noise pollution is adopted. By regarding the observations on several evaluation quantities: $L_{\text {Aeq.T }}$ and $L_{A 50, T}$, measured by use of sound level meter with the frequency characteristic of $\mathrm{A}$ weight and the time interval $T=1 \mathrm{~min}$. under existence of a background noise, as observations $y_{k}$, and regarding the evaluation quantities: $L_{\text {Aeq }, T}$ and $L_{A 50, T}(T=1 \mathrm{~min})$ for only the specific signal as the state variables $x_{k}$, the fluctuation of $x_{k}$ is estimated on the basis of $y_{k}$.

By dividing the fluctuation ranges of $x_{k}$ and $y_{k}$ into 3 fuzzy regions, $A_{1}, B_{1}$ : "low level", $A_{2}, B_{2}$ : "middle level", and: $A_{3}, B_{3}$ "high level", (1) is expressed as follows:

$$
\begin{aligned}
& \text { Rule 1: IF } x_{k} \text { is } A_{1} \text { THEN } y_{k} \text { is } B_{1} \\
& \text { Rule 2: IF } x_{k} \text { is } A_{2} \text { THEN } y_{k} \text { is } B_{2} \\
& \text { Rule 3: IF } x_{k} \text { is } A_{3} \text { THEN } y_{k} \text { is } B_{3} .
\end{aligned}
$$

Furthermore, (2) is expressed as follows:

$$
y_{k}=\frac{\bar{y}_{1} \mu_{A_{1}}\left(x_{k}\right)+\bar{y}_{2} \mu_{A_{2}}\left(x_{k}\right)+\bar{y}_{3} \mu_{A_{3}}\left(x_{k}\right)}{\mu_{A_{1}}\left(x_{k}\right)+\mu_{A_{2}}\left(x_{k}\right)+\mu_{A_{3}}\left(x_{k}\right)} .
$$

In the above formula, the three parameters are decided as:

$$
\bar{y}_{1}=\mu_{y}-3 \sigma_{y}, \bar{y}_{2}=\mu_{y}, \bar{y}_{3}=\mu_{y}+3 \sigma_{y}
$$

where $\mu_{y}$ and $\sigma_{y}$ are mean and standard deviation of $y_{k}$. Furthermore, by determining the parameters of (28) so as to express the whole distribution of actual measured data of $x_{k}$ as precisely as possible, the membership functions in Figures 1 and 2 are obtained for $L_{\text {Aeq,T }}$ and $L_{A 50, T}$.

For comparison, the previously reported method $[4,5]$ without considering fuzzy theory is also applied to the observed data after introducing a linear system model:

$$
\begin{aligned}
& y_{k}=a_{k} x_{k}+b_{k} v_{k}, \\
& x_{k+1}=F x_{k}+G u_{k}, a_{k+1}=a_{k}, b_{k+1}=b_{k}
\end{aligned}
$$

In (33) and (34), $a_{k}$ and $b_{k}$ are unknown parameters to be estimated simultaneously with the state variable $x_{k}$. Furthermore, $v_{k}$ and $u_{k}$ are random noises with mean 0 and variance 1 . Tow parameters $F$ and $G$ are estimated by use of auto-correlation technique [4]. The estimation results for $L_{A e q, 1 \min }$ and $L_{A 50,1 \min }$ of the road traffic noise by observing $L_{\text {Aeq, Imin }}$ and $L_{A 50,1 \text { min }}$ under existence of a background noise are shown in Figures $\mathbf{3}$ and $\mathbf{4}$. The proposed method estimates precisely the fluctuation of the state variables. On the other hand, there are great

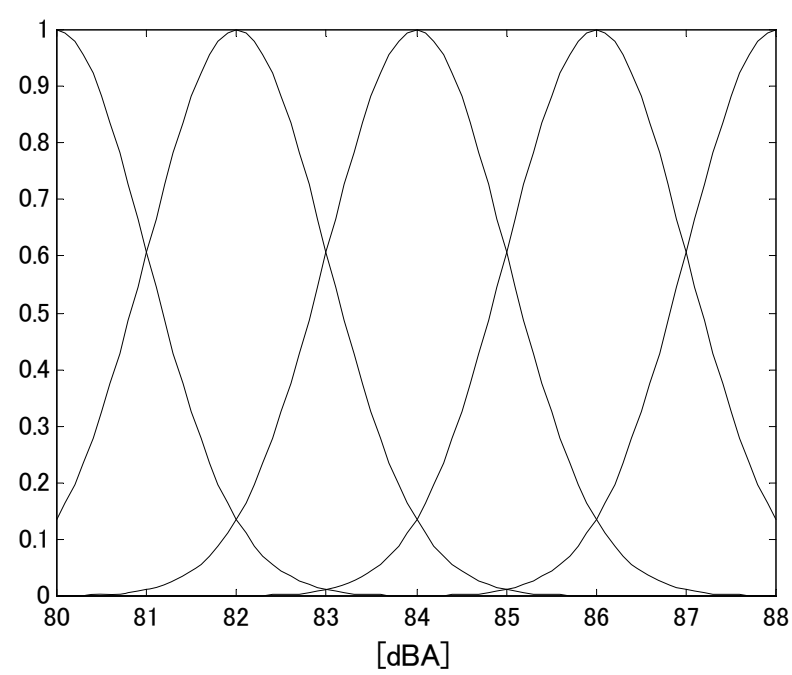

Figure 1. Membership function for $L_{\text {Aeq, } 1 \text { min }}$.

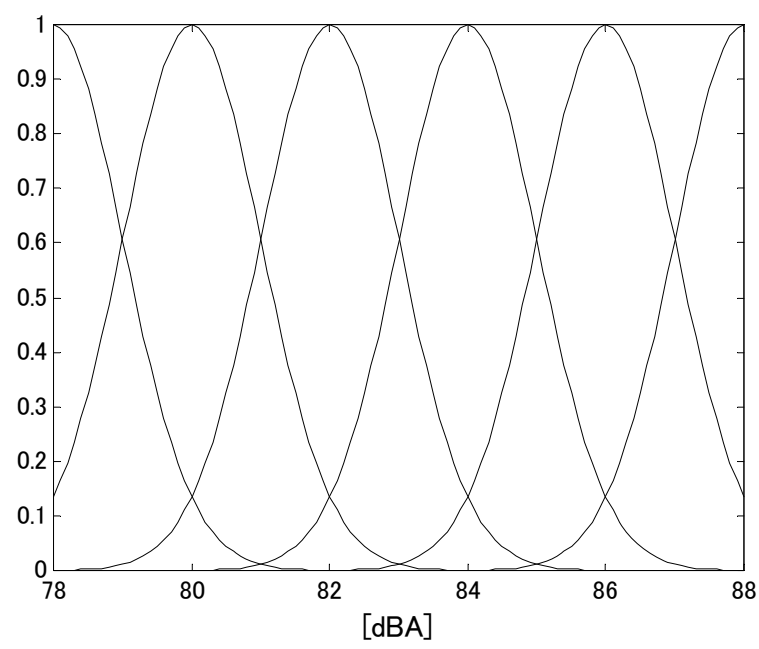

Figure 2. Membership function for $L_{A 50,1 \mathrm{~min}}$.

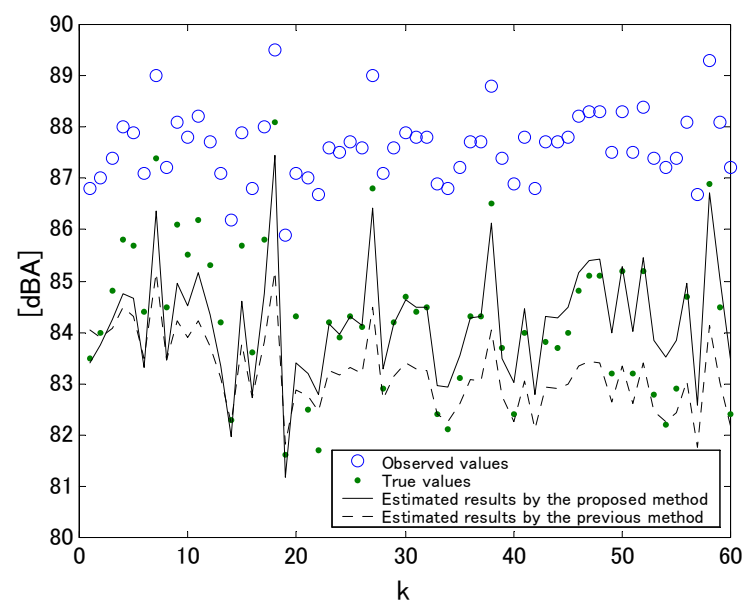

Figure 3. A comparison between the proposed method and the previous method for state estimation results of $L_{\text {Aeq, } 1 \mathrm{~min}}$ for the road traffic noise based on the observation of $L_{\text {Aeq, } 1 \text { min }}$ under existence of a background noise. 


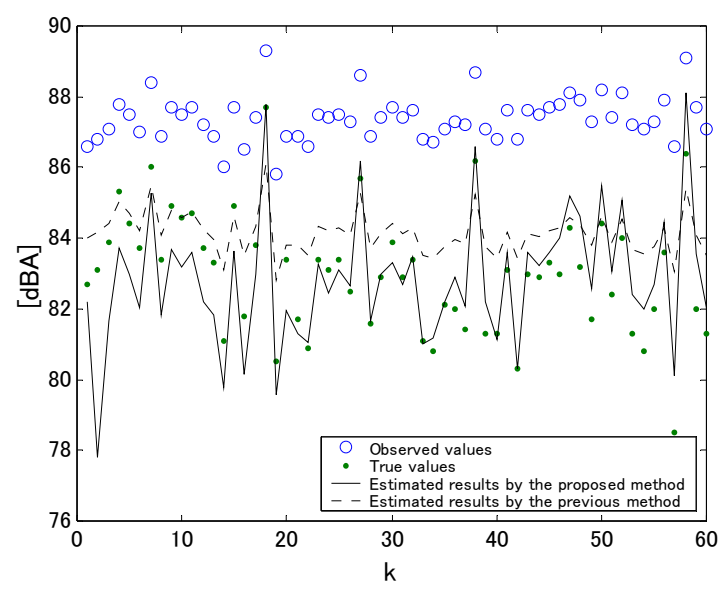

Figure 4. A comparison between the proposed method and the previous method for of state estimation results of $L_{A 50,1 \mathrm{~min}}$ for the road traffic noise based on the observation of $L_{A 50,1 \mathrm{~min}}$ under existence of a background noise.

discrepancies between the true values and the estimates based on the linear system of (33) and (34). The squared sum of the estimation error is shown in Table 1. It is obvious that the proposed method based on the introduction of fuzzy theory shows more accurate estimation than the results based on the usual linear system model like (33) and (34).

Next, as one of reasonable methods for determining the three parameters in (31), by introducing an unknown parameter $\beta$ in (32) as:

$$
\bar{y}_{1}=\mu_{y}-\beta \sigma_{y}, \bar{y}_{2}=\mu_{y}, \bar{y}_{3}=\mu_{y}+\beta \sigma_{y}
$$

and focusing our attention on the Bayes' theorem for the conditional pdf of $x_{k}, \alpha_{k}$ and $\beta_{k}$ :

$$
P\left(x_{k}, \alpha_{k}, \beta_{k} \mid Y_{k}\right)=\frac{P\left(x_{k}, \alpha_{k}, \beta_{k}, y_{k} \mid Y_{k-1}\right)}{P\left(y_{k} \mid Y_{k-1}\right)},
$$

after introducing a dynamical model for $\beta$ :

$$
\beta_{k+1}=\beta_{k}
$$

an estimation algorithm for the state variable can be derived through the same calculation process as (7). The estimation results by the proposed method with introduction of the unknown parameter $\beta$ are shown in Figures 5 and 6 . In general, the results by the proposed method using (35) estimate precisely the peak values of fluctuation than the results by the method using (32). A comparison between the squared sum of the estimation error in two cases is also shown in Table 1. The estimated results by the algorithm with unknown parameter $\beta$ show more accurate estimation than the results based on the method with the fixed value of $\beta$.

Furthermore, the proposed method with flexibility is applied to estimate $L_{\text {Aeq, } 1 \text { min }}$ of the road traffic noise based on the observation of $L_{A 50,1 \text { min }}$ under existence of

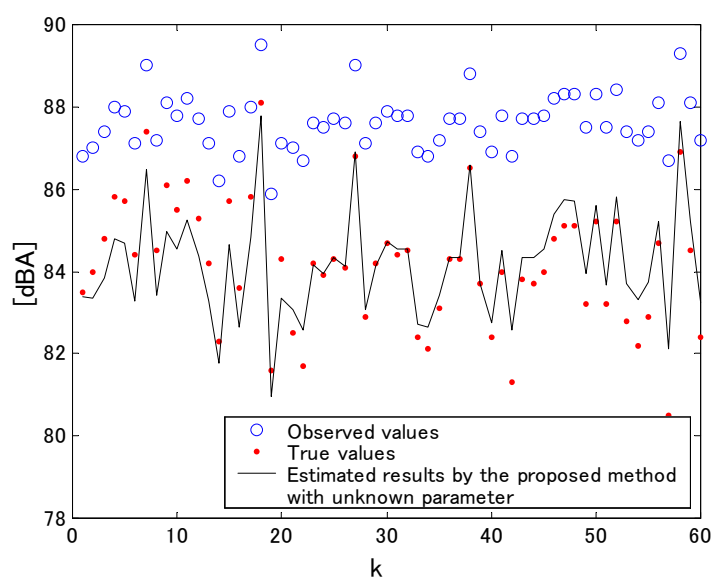

Figure 5. A state estimation result of $L_{A e q, 1 m i n}$ for the road traffic noise based on the observation of $L_{A e q, 1 \mathrm{~min}}$ by the proposed method with unknown parameter.

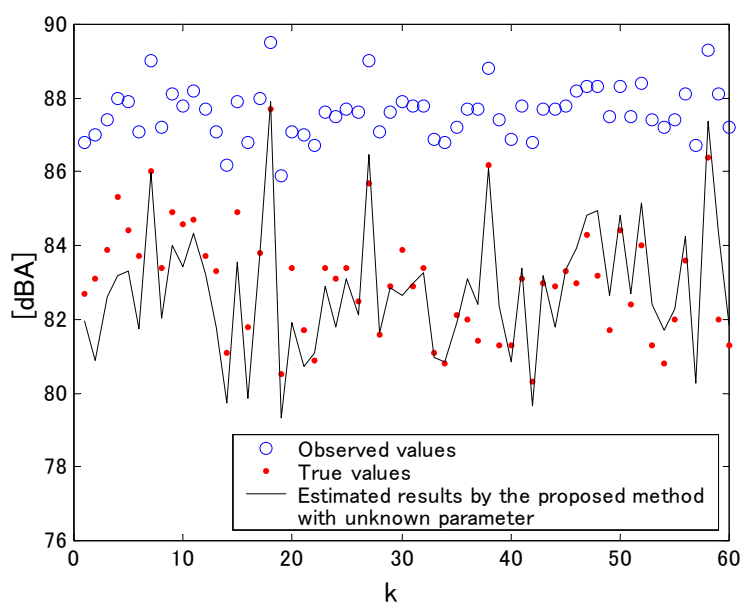

Figure 6. A state estimation result of $L_{A 50,1 \min }$ for the road traffic noise based on the observation of $L_{A 50,1 \mathrm{~min}}$ by the proposed method with unknown parameter.

Table 1. Root-mean squared error of the estimation (in dBA).

\begin{tabular}{ccccc}
\hline $\begin{array}{c}\text { State } \\
\text { Variable }\end{array}$ & Observation & $\begin{array}{c}\text { Proposed Method } \\
\text { with Fixed } \\
\text { Parameter }\end{array}$ & $\begin{array}{c}\text { Proposed } \\
\text { Method with } \\
\text { Unknown } \\
\text { Parameter }\end{array}$ & $\begin{array}{c}\text { Previous } \\
\text { Method }\end{array}$ \\
\hline$L_{\text {Aeq, } 1 \min }$ & $L_{\text {Aeq, } 1 \mathrm{~min}}$ & 0.749 & 0.718 & 1.31 \\
$L_{A 50,1 \min }$ & $L_{A 50,1 \min }$ & 1.20 & 1.07 & 1.61 \\
\hline
\end{tabular}

the background noise as a trial. It must be noticed that the observation and the specific signal to be estimated are different evaluation quantities in this case. Figure 7 shows a comparison between the proposed method and the previous method, and the estimated result by use of the proposed method with unknown parameter is shown in Figure 8. The root mean squared error of the estimation is shown in Table 2. From these results, the effectiveness of the proposed method has been confirmed even 


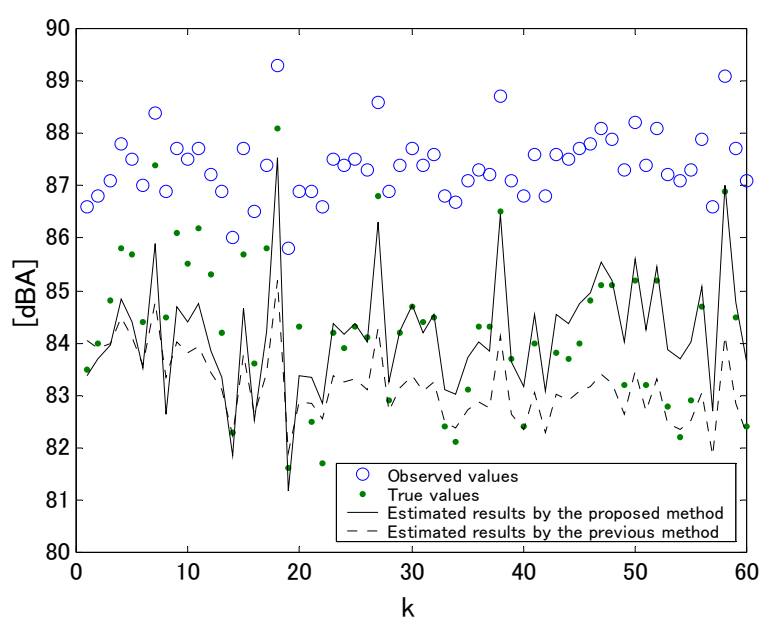

Figure 7. A comparison between the proposed method and the previous method for state estimation results of $L_{\text {Aeq,1min }}$ for the road traffic noise based on the observation of $L_{A 50,1 \mathrm{~min}}$ under existence of a background noise.

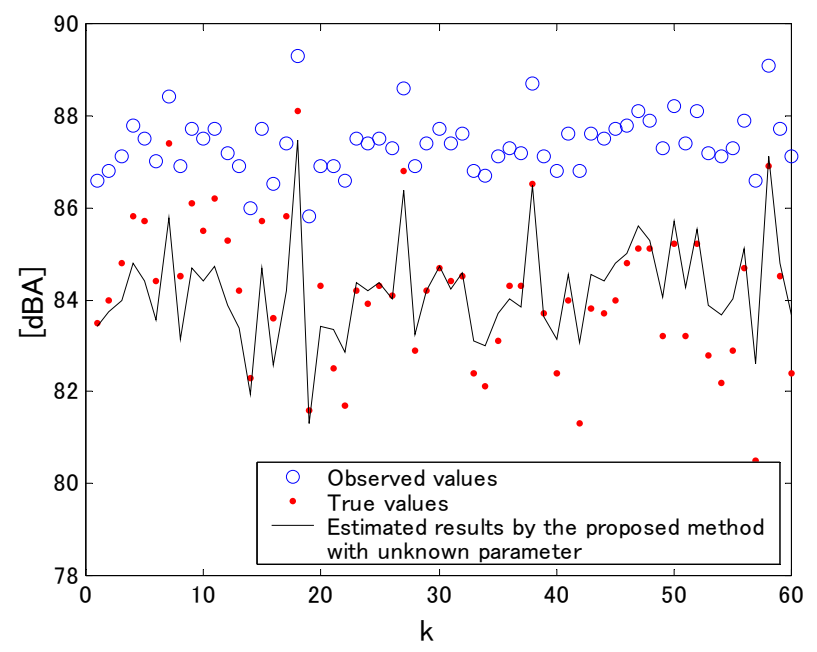

Figure 8. A state estimation result of $L_{A e q, 1 \mathrm{~min}}$ for the road traffic noise based on the observation of $L_{A 50,1 \min }$ by the proposed method with unknown parameter.

Table 2. Root-mean squared error of the estimation in Figures 7 and 8 (in dBA).

\begin{tabular}{ccccc}
\hline $\begin{array}{c}\text { State } \\
\text { Variable }\end{array}$ & Observation & $\begin{array}{c}\text { Proposed } \\
\text { Method with } \\
\text { Fixed } \\
\text { Parameter }\end{array}$ & $\begin{array}{c}\text { Proposed } \\
\text { Method with } \\
\text { Unknown } \\
\text { Parameter }\end{array}$ & $\begin{array}{c}\text { Previous } \\
\text { Method }\end{array}$ \\
\hline$L_{\text {Aeq, } 1 \text { min }}$ & $L_{\text {Aeq, } 1 \text { min }}$ & 0.896 & 0.875 & 1.38 \\
\hline
\end{tabular}

in the case of observation of different evaluation quantity.

\section{Conclusions}

In this study, a practical state estimation method for the sound environment system with uncertainty has been theoretically proposed by introducing the fuzzy inference.
More specifically, after regarding the relationship between the state variable and observed evaluation quantities under existence of a background noise as a sound environment system with unknown observation mechanism, a recursive estimation algorithm for evaluation quantities of the specific signal has been derived. Furthermore, by applying the proposed method to the actually observed data of road traffic noise in the sound environment, the effectiveness of the theory has been confirmed experimentally too.

There are two points to be considered for obtaining improved estimations, as compared with the previous method. The first point is that our proposed method introduces fuzzy theory on the basis of fundamental principles of probability theory. More specifically, based on the Bayes' theorem which is a normative axiom of probability theory, by introducing fuzzy inference for the uncertainty of the objective system, the generalization ability can be enhanced. The second point is that we can express the fuzziness of phenomena in a form reflecting the vague fluctuation around a true value. That is, by estimating simultaneously the parameter of the membership function with the state variable based on the observation data, the new state estimation algorithm has been derived.

The proposed approach is quite different from the traditional standard approach. Thus, it is still at an early stage of study, and there are left a number of practical problems to be continued in the future, starting from the result of the basic study in this paper. Some of the problems are the following.

1) The proposed method should be applied to the other actual data of sound environment, and its practical usefulness should be verified in each actual situation. For example, the proposed estimation method can be applied to the actual sound insulation systems with complex structure. By applying the proposed method to the input and output data of sound insulation systems, the system parameters reflecting the sound insulation properties can be estimated.

2) The proposed theory should be further extended into more complicated situation with multi-signal sources. In the acoustical engineering field, it is very important to separate only the objective specific signal from many other signals based on the compound observation in the actual situation in the presence of external noise. A future challenge is the development of an estimation method under the multi-signal sources, on the basis of the proposed method.

3) The theory should be developed to find more precise estimation methods for the state variables by introducing a hybrid method of fuzzy theory and statistical methods. More specifically, by taking the higher order correlation information between the state variables and 
the observation into consideration in the fuzzy theory, a new type of state estimation method can be proposed.

\section{REFERENCES}

[1] R. E. Kalman and R. S. Bucy, "New Results in Linear Filtering and Prediction Theory," Transactions of ASME, Series D, Journal of Basic Engineering, Vol. 83, No. 1, 1961, pp. 95-108. doi:10.1115/1.3658902

[2] R. E. Kalman, "A New Approach to Linear Filtering and Prediction Problems," Transactions of ASME, Series D, Journal of Basic Engineering, Vol. 82, No. 1, 1960, pp. 35-45. doi:10.1115/1.3662552

[3] H. J. Kushner, "Approximations to Optimal Nonlinear Filter," IEEE Transactions on Automatic Control, Vol. 12, No. 5, 1967, pp. 546-556. doi:10.1109/TAC.1967.1098671

[4] M. Ohta and H. Yamada, "New Methodological Trials of Dynamical State Estimation for the Noise and Vibration Environmental System," Acustica, Vol. 55, No. 4, 1984, pp. 199-212.

[5] A. Ikuta and M. Ohta, "A State Estimation Method of Impulsive Signal Using Digital Filter under the Existence of External Noise and Its Application to Room Acoustics," IEICE Transactions on Fundamentals of Electronics, Communications and Computer Sciences, Vol. E75-A, No. 8, 1992, pp. 988-995.

[6] A. Ikuta and M. Ohta, "A Cancellation Method for Back- ground Noise in Sound and Electromagnetic Environments by Using a Digital Filter for Power State Variables," International Journal of Acoustics and Vibration, Vol. 8, No. 2, 2003, pp. 104-110.

[7] S. Julier and J. Uhlmann, "Unscented Filtering and Nonlinear Estimation," Proceedings of the IEEE, Vol. 92, No. 3, 2004, pp. 401-421. doi:10.1109/JPROC.2003.823141

[8] G. Kitagawa, "Monte Carlo Filter and Smoother for NonGaussian Nonlinear State Space Models," Journal of Computational and Graphical Statistics, Vol. 5, No. 1, 1996, pp. 1-25.

[9] H. Orimoto and A. Ikuta, "Adaptive Method for State Estimation of Sound Environment System with Uncertainty and Its Application to Psychological Evaluation," Intelligent Information Management, Vol. 2, No. 3, 2010, pp. 212-219. doi:10.4236/iim.2012.23025

[10] L. X. Wang and J. M. Mendel, "Fuzzy Basic Functions, Universal Approximation, and Orthogonal Least-Squares Learning," IEEE Transactions on Neural Networks, Vol. 3, No. 5, 1992, pp. 807-814. doi:10.1109/72.159070

[11] M. Ohta and T. Koizumi, "General Statistical Treatment of the Response of a Non-Linear Rectifying Device to a Stationary Random Input," IEEE Transactions on Information Theory, Vol. 14, No. 4, 1968, pp. 595-598. doi:10.1109/TIT.1968.1054178

[12] L. X. Wang, "Fuzzy Systems and Control: Design and Stability Analysis,” PTR Prentice Hall, New York, 1994. 